\title{
LIMIT CYCLES OF PIECEWISE LINEAR DIFFERENTIAL SYSTEMS WITH THREE ZONES AND NO SYMMETRY
}

\author{
JAUME LLIBRE ${ }^{1}$, ENRIQUE PONCE $^{2}$ AND CLÀUDIA VALLS ${ }^{3}$
}

\begin{abstract}
Some techniques for proving the existence and uniqueness of limit cycles for smooth differential systems, are extended to continuous piecewiselinear differential systems. Then we obtain new results on the existence and uniqueness of limit cycles for systems with three linearity zones and without symmetry. We also reprove existing results of systems with two linearity zones giving shorter and clearer proofs.
\end{abstract}

\section{Introduction AND StATEMEnt of the MAIN RESUlts}

One of the most interesting problems in the qualitative theory of planar differential systems is the study of their limit cycles, see for instance the books $[35,36]$. This problem restricted to the planar polynomial differential equations is the famous second part of the 16-th Hilbert problem [16]. Due to the fact this Hilbert problem becomes up to now intractable (see [19, 23]), Smale in [33] propose to study it restricted to the polynomial Liénard differential systems.

For smooth Liénard systems there are many results on the non-existence, existence and uniqueness of limit cycles, see for instance $[1,6,8,14,21,24,29,34,36]$. Going beyond the smooth case a natural step is to allow non-smoothness while keeping the continuity, as has been done in some recent works $[10,17,18,25]$. In a further step, other authors have considered a line of discontinuity in the vector field defining the planar system, see [15, 26, 37].

In this work we will study the limit cycles of systems of the form

$$
\frac{d \mathbf{x}}{d s}=\dot{\mathbf{x}}=A \mathbf{x}+\phi(\mathbf{k} \cdot \mathbf{x}) \mathbf{b},
$$

where $A$ is a $2 \times 2$ real matrix and $\mathbf{x}, \mathbf{k}$ and $\mathbf{b}$ are in $\mathbb{R}^{2}$ and $\mathbf{k} \cdot \mathbf{x}$ denotes the usual inner product between the vectors $\mathbf{k}$ and $\mathbf{x}$. These systems are very important in direct control and other areas, see for instance [5, 22, 30]. The divergence from linearity of these systems results from the presence of the characteristic function $\phi$, here given by

$$
\phi(x)= \begin{cases}m_{L}(x+u)-u & \text { for } x \leq-u, \\ x & \text { for }-u \leq x \leq v, \\ m_{R}(x-v)+v & \text { for } x \geq v,\end{cases}
$$

where $u$ and $v$ are positive, and $u, v, m_{L}, m_{R} \in \mathbb{R}$ are fixed parameters. In the case of control systems the objective of the function $\phi$ is to improve the asymptotic stability

\footnotetext{
2010 Mathematics Subject Classification. Primary 34C25. Secondary 34A34.

Key words and phrases. Nonlinear control systems, periodic orbits, limit cycles, Liénard piecewise linear differential systems.
} 
behavior of the equilibrium located at the origin, and its nonlinear character comes typically from saturation effects. In other cases the function $\phi$ naturally arises as the characteristic of a nonlinear device.

We introduce the parameters

$$
t=\operatorname{trace} A, \quad d=\operatorname{det} A, \quad T=\operatorname{trace} B \quad \text { and } \quad D=\operatorname{det} B,
$$

where $B=A+\mathbf{b k}^{t}$.

As we shall see later on the piecewise linear differential systems (1) can be written as the Liénard piecewise linear differential systems

$$
\frac{d x}{d \tau}=x^{\prime}=F(x)-y, \quad \frac{d y}{d \tau}=y^{\prime}=g(x),
$$

where

$$
F(x)= \begin{cases}T_{L}(x+u)-T_{C} u & \text { if } x \leq-u, \\ T_{C} x & \text { if }-u \leq x \leq v, \\ T_{R}(x-v)+T_{C} v & \text { if } x \geq v\end{cases}
$$

and

$$
g(x)= \begin{cases}\ell(x+u)-u & \text { if } x \leq-u \\ x & \text { if }-u \leq x \leq v, \\ r(x-v)+v & \text { if } x \geq v\end{cases}
$$

where the constants $u$ and $v$ are the same that appear in the definition of characteristic function, and $\tau=-\sqrt{D} s$.

We shall see in section 3 that the differential systems (1) and (2) are equivalent if $D>0$, i.e. there exists a change of variables which transforms one system into the other.

The main goal of this paper is to study the number of limit cycles of systems (1), or equivalently of systems (2) when $D>0$. In the case that these systems are symmetric with respect the origin of coordinates, i.e.

$$
u=v, \quad m_{L}=m_{R}, \quad T_{L}=T_{R}, \quad \ell=r .
$$

the study of their limit cycles is done, see $[2,10,11,12,27]$, and for a complete analysis the book [28].

In this paper we shall study the limit cycles of systems (2) or (1) when $D>0$ in the non-symmetric case with respect to the origin of coordinates. Of course, as a particular case we shall get the symmetric case. The first main result in this direction is the following theorem that characterizes the number of limit cycles when there are only two linearity zones.

We need the following definitions. Let

$$
Y_{ \pm}^{r}= \pm v \exp \left( \pm \gamma \theta_{ \pm}\right) \sqrt{\left(\sigma-\mu_{ \pm}\right)^{2}+\omega^{2}}
$$

where, provided that $T_{C} \in(-2,2)$ and $r<0$, we take

$$
\gamma=\frac{T_{C}}{\sqrt{4-T_{C}^{2}}}, \quad \mu_{ \pm}=\frac{T_{R} \pm \sqrt{T_{R}^{2}-4 r}}{2 r}, \quad \sigma=\frac{T_{C}}{2}, \quad \omega=\frac{\sqrt{4-T_{C}^{2}}}{2},
$$


and

Moreover, let

$$
\theta_{ \pm}=2 \arctan \left(\frac{\omega}{\sqrt{\left(\sigma-\mu_{ \pm}\right)^{2}+\omega^{2}} \pm\left(\sigma-\mu_{ \pm}\right)}\right)
$$

$$
Y_{ \pm}^{\ell}= \pm u \exp \left( \pm \gamma \psi_{ \pm}\right) \sqrt{\left(\sigma-\delta_{ \pm}\right)^{2}+\omega^{2}}
$$

where it is assumed $\ell<0$, and we take

$$
\delta_{ \pm}=\frac{T_{L} \pm \sqrt{T_{L}^{2}-4 \ell}}{2 \ell} \quad \text { and } \quad \psi_{ \pm}=2 \arctan \left(\frac{\omega}{\sqrt{\left(\sigma-\delta_{ \pm}\right)^{2}+\omega^{2}} \pm\left(\sigma-\delta_{ \pm}\right)}\right) .
$$

Theorem 1. Consider the differential systems (2) with only two linearity zones, where $T_{L}=T_{C}$ and $\ell=1$. Assume $T_{C} \neq 0$. Then the following statements hold.

(a) Two necessary conditions for the existence of periodic orbits are $\left|T_{C}\right|<2$ and $T_{C} T_{R}<0$.

(b) Assume that $\left|T_{C}\right|<2$ and $T_{C} T_{R}<0$. Then system (2) has a periodic orbit

(b.1) when $T_{C}>0$ and $r>0$ if and only if $T_{C}+T_{R} / \sqrt{r}<0$;

(b.2) when $T_{C}<0$ and $r>0$ if and only if $T_{C}+T_{R} / \sqrt{r}>0$;

(b.3) when $T_{C}>0$ and $r<0$ if and only if $e^{\pi \gamma} Y_{+}^{r}+Y_{-}^{r}<0$;

(b.4) when $T_{C}<0$ and $r<0$ if and only if $e^{\pi \gamma} Y_{+}^{r}+Y_{-}^{r}>0$.

Moreover in all cases that the origin is surrounded by a limit cycle which is unique, stable if $T_{C}>0$ and unstable if $T_{C}<0$.

The proof of Theorem 1 is given in section 5. Its dual result, whose proof is similar and we will not provide it, is the following.

Theorem 2. Consider the differential systems (2) with only two linearity zones, where $T_{R}=T_{C}$ and $r=1$. Assume $T_{C} \neq 0$. Then the following statements hold.

(a) Two necessary conditions for the existence of periodic orbits are $\left|T_{C}\right|<2$ and $T_{L} T_{C}<0$.

(b) Assume that $\left|T_{C}\right|<2$ and $T_{L} T_{C}<0$. Then system (2) has a periodic orbit (b.1) when $T_{C}>0$ and $\ell>0$ if and only if $T_{C}+T_{L} / \sqrt{\ell}<0$;

(b.2) when $T_{C}<0$ and $\ell>0$ if and only if $T_{C}+T_{L} / \sqrt{\ell}>0$;

(b.3) when $T_{C}>0$, and $\ell<0$ if and only if $e^{\pi \gamma} Y_{+}^{\ell}+Y_{-}^{\ell}<0$;

(b.4) when $T_{C}<0$, and $\ell<0$ if and only if $e^{\pi \gamma} Y_{+}^{\ell}+Y_{-}^{\ell}>0$.

Moreover in all cases that the origin is surrounded by a limit cycle, this is unique, stable if $T_{C}>0$ and unstable if $T_{C}<0$.

Theorems 1 and 2 improve and extend cases studied in [9]. Here we give a shorter and clear proof using the techniques developed in [25]. In [25] the authors proved Theorem 1 with the additional hypothesis that systems (2) have only one equilibrium point, which is the origin and with $T_{C}>0$. This is equivalent to state that $r \geq 0$ in the case $T_{L}=T_{C}$ and $\ell=1$, and that $\ell \geq 0$ in the case $T_{R}=T_{C}$ and $r=1$. We extend Theorem 2 of [25] to cover all cases.

We remark that if we consider for instance the case of Theorem 1 (b.1) and we allow $T_{C}$ to vanish, then we have a center at the origin, its periodic annulus is bounded by the line $x=v$. If we perturb this degenerate situation by taking $T_{C}>0$ and small, then the necessary and sufficient condition of the statement is automatically fulfilled, so that we conclude that such a perturbation implies a 
bifurcation of a limit cycle from the periodic annulus. This bifurcation and the analogous for the other statements of Theorems 1 and 2 are quantitatively studied in a more general setting in [32].

In the case of three linearity zones our main result is the following.

Theorem 3. Consider the differential systems (2) and assume $T_{C}>0$. Then the following statements hold.

(a) A necessary condition for the existence of periodic orbits is that the traces $T_{L}, T_{C}, T_{R}$ have not the same sign.

(b) If $T_{R}, T_{L}<0$, and $r, \ell \geq 0$, then the origin is surrounded by a limit cycle which is unique and stable.

(c) If $T_{R}$ and $T_{L}$ have the same sign and opposite to the sign of $T_{C}$, then the origin is surrounded by at most one limit cycle, that if it exists is stable.

(d) If $T_{R}$ and $T_{C}$ have the same sign and opposite to the sign of $T_{L}$, then the origin is surrounded by at most one limit cycle, that if it exists is stable.

(e) If $T_{L}$ and $T_{C}$ have the same sign and opposite to the sign of $T_{R}$, then the origin is surrounded by at most one limit cycle, that if it exists is stable.

Theorem 3 is proved in section 6 . Its immediate dual result, which is given without proof, is the following.

Theorem 4. Consider the differential systems (2) and assume $T_{C}<0$. Then the following statements hold.

(a) A necessary condition for the existence of periodic orbits is that the traces $T_{L}, T_{C}, T_{R}$ have not the same sign.

(b) If $T_{R}, T_{L}>0$, and $r, \ell \geq 0$, then the origin is surrounded by a limit cycle which is unique and unstable.

(c) If $T_{R}$ and $T_{L}$ have the same sign and opposite to the sign of $T_{C}$, then the origin is surrounded by at most one limit cycle, that if it exists is unstable.

(d) If $T_{R}$ and $T_{C}$ have the same sign and opposite to the sign of $T_{L}$, then the origin is surrounded by at most one limit cycle, that if it exists is unstable.

(e) If $T_{L}$ and $T_{C}$ have the same sign and opposite to the sign of $T_{R}$, then the origin is surrounded by at most one limit cycle, that if it exists is unstable.

Theorem 3 is Theorem 1 of [25] in the case that the sign of $T_{R}$ and $T_{L}$ are equal and different from the sign of $T_{C}$ and under the additional assumption that $T_{C}>0$ and the origin is the unique equilibrium point of the system, i.e. that $r \geq 0$ and $\ell \geq 0$. Here we extend the result of [25] covering all the cases when the sign of $T_{R}$ and $T_{L}$ are equal and different from the sign of $T_{C}$. Moreover we also study the cases in which either $T_{C}$ and $T_{R}$ have the same sign and $T_{L}$ has different sign, or $T_{C}$ and $T_{L}$ have the same sign and $T_{R}$ has different sign. More precise information on the relations between Theorem 3 and Theorem 1 of [25] is given at the beginning of section 6 .

In the next section we show the usefulness of this kind of results in a relevant problem of nonlinear electronics. 


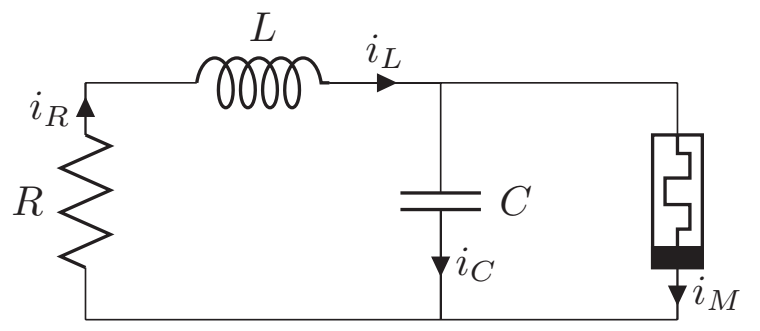

FiguRE 1. The simple oscillator with one memristor analyzed in this section. Note that the negative value considered for $R$ makes it the only active element in the circuit.

\section{Application to the study of a simple oscillator with one MEMRISTOR}

Following [4] memristors are two-terminal electronic passive devices for which a nonlinear relationship links charge and flux [3]. They seem to be at the basis of future generation oscillatory associative and dynamic memories; as another important feature, nanoscale memristors have potential to reproduce the behavior of biological synapses. Here we apply our previous results to the analysis of an elementary oscillator endowed with one flux-controlled memristor, see Figure 1 and $[20]$.

In the shown circuit the values of $L$ and $C$ for the impedance and capacitance are positive constants, while the resistor $R$ has a negative value. From Kirchoff's laws we see that

$$
\begin{aligned}
i_{R}(\tau)-i_{L}(\tau) & =0, \\
i_{L}(\tau)-i_{C}(\tau)-i_{M}(\tau) & =0, \\
v_{R}(\tau)+v_{L}(\tau)+v_{C}(\tau) & =0, \\
v_{C}(\tau)-v_{M}(\tau) & =0,
\end{aligned}
$$

where $v, i$ stand for the voltage and current, respectively, across the corresponding element of the circuit.

Integrating with respect to time the above equations, and assuming as in [4] that all the initial conditions are zero, we get

$$
\begin{aligned}
q_{R}(\tau)-q_{L}(\tau) & =0 \\
q_{L}(\tau)-q_{C}(\tau)-q_{M}(\tau) & =0 \\
\varphi_{R}(\tau)+\varphi_{L}(\tau)+\varphi_{C}(\tau) & =0 \\
\varphi_{C}(\tau)-\varphi_{M}(\tau) & =0
\end{aligned}
$$

where $q$ and $\varphi$ stand respectively for the charge and flux associated to each element. 
We denote by $f_{M}$ the flux-charge characteristic of the memristor and, after recalling the constitutive equations of the bipoles, namely

$$
\begin{aligned}
\varphi_{R}(\tau) & =R q_{R}(\tau), \\
\varphi_{L}(\tau) & =L \frac{d}{d \tau} q_{L}(\tau), \\
q_{C}(\tau) & =C \frac{d}{d \tau} \varphi_{C}(\tau), \\
q_{M}(\tau) & =f_{M}\left(\varphi_{M}(\tau)\right),
\end{aligned}
$$

we arrive at the equations

$$
\begin{aligned}
\frac{d}{d \tau} \varphi_{C}(\tau) & =\frac{1}{C} q_{C}(\tau)=\frac{1}{C}\left[q_{L}(\tau)-q_{M}(\tau)\right] \\
\frac{d}{d \tau} q_{L}(\tau) & =\frac{1}{L} \varphi_{L}(\tau)=\frac{1}{L}\left[-\varphi_{C}(\tau)-\varphi_{R}(\tau)\right]
\end{aligned}
$$

We denote the state variables by $x_{1}=\varphi_{C}(\tau)$ and $x_{2}=q_{L}(\tau)$, and using (5) and (8), to write $\varphi_{R}(\tau)=R q_{R}(\tau)=R q_{L}(\tau)$ and $q_{M}(\tau)=f_{M}\left(\varphi_{M}(\tau)\right)=f_{M}\left(\varphi_{C}(\tau)\right)$, we have the differential system

$$
\begin{aligned}
\frac{d x_{1}}{d \tau} & =\frac{1}{C}\left[-f_{M}\left(x_{1}\right)+x_{2}\right] \\
\frac{d x_{2}}{d \tau} & =\frac{1}{L}\left[-x_{1}-R x_{2}\right]
\end{aligned}
$$

Instead of the symmetric piecewise linear function considered in [20] and [4], we adopt here a more general model for the nonlinear flux-charge characteristic of the memristor, namely

$$
f_{M}(x)= \begin{cases}b_{L}(x+u)-a u & \text { for } x \leq-u \\ a x & \text { for }-u \leq x \leq v \\ b_{R}(x-v)+a v & \text { for } x \geq v\end{cases}
$$

So we can apply our results to symmetric and non-symmetric oscillators. A rescaling of the time by putting $\tau=C s$ and the use of the positive parameters

$$
G=-\frac{1}{R}, \quad \nu=-\frac{R C}{L}
$$

brings the system to

$$
\begin{aligned}
& \frac{d x_{1}}{d s}=-f_{M}\left(x_{1}\right)+x_{2}, \\
& \frac{d x_{2}}{d s}=-\nu\left(G x_{1}-x_{2}\right),
\end{aligned}
$$

which can be written as a system (1) with

$$
A=\left(\begin{array}{cc}
0 & 1 \\
-G \nu & \nu
\end{array}\right), \quad \mathbf{k}=\left(\begin{array}{l}
1 \\
0
\end{array}\right), \quad \mathbf{b}=\left(\begin{array}{l}
a \\
0
\end{array}\right)
$$

and with a $\phi$ such that $m_{L}=b_{L} / a, m_{R}=b_{R} / a$. 
Now it is very easy to write the system in the Liénard form of Lemma 7 but with the time reversed, by doing the change of variables $x=x_{1}, y=\nu x_{1}-x_{2}$, getting

$$
\begin{aligned}
& \frac{d x}{d s}=\nu x-f_{M}(x)-y, \\
& \frac{d y}{d s}=\nu\left[G x-f_{M}(x)\right] .
\end{aligned}
$$

A natural assumption is to take the determinant in the central region is to be positive, which requires $a<G$. We assume such condition in the sequel, and after introducing for convenience a positive constant $\omega$, we have

$$
D=\nu(G-a)=\omega^{2}>0 .
$$

Therefore under the above assumption, using the transformation given in the proof of Proposition 8 without reversing the time, namely the change $(x, y, s) \mapsto(X, Y, \tau)$ given by

$$
X=x, \quad Y=\frac{y}{\sqrt{D}}=\frac{y}{\omega} \text { and } \quad \tau=\omega s,
$$

we get that system (9)-(10) can be written as (2), satisfying (3)-(4) with

and

$$
T_{L}=\frac{\nu-b_{L}}{\omega}, \quad T_{C}=\frac{\nu-a}{\omega}, \quad T_{R}=\frac{\nu-b_{R}}{\omega},
$$

$$
\ell=\frac{G \nu-b_{L} \nu}{G \nu-a \nu}=\frac{G-b_{L}}{G-a}, \quad r=\frac{G \nu-b_{R} \nu}{G \nu-a \nu}=\frac{G-b_{R}}{G-a} .
$$

We are now able to apply in a convenient way our previous results, without trying to reproduce all the statements but only the most significant cases. We consider first the case of non-symmetric memristors with three linear zones, getting from a direct application of Theorem 3(b) the following result. Thus we consider the case $T_{C}>0, T_{L}, T_{R}<0$ with $\ell, r>0$.

Proposition 5. Assuming $\nu<G$, that is $R^{2} C<L$, and the additional hypotheses $a<\nu, \nu<b_{L} \leq G$ and $\nu<b_{R} \leq G$, the circuit exhibits stable oscillations, corresponding to a stable limit cycle in the phase plane state variables.

If we now consider rather non-symmetric memristors, namely those with a characteristic with only two zones, from Theorem 1(b.1), we can deduce the following result. Note that this case is also relevant when the system has indeed three zones but the lack of symmetry allows to discard one zone whenever we can reasonably assume that the periodic orbit will only use two zones. Thus, we take $b_{L}=a$ so that $\ell=1$, and we require that $0<T_{C}<2$ (unstable focus at the origin) and $T_{R}<0$, so to fulfill the necessary conditions for oscillation. The condition $T_{C}<2$ translates after some elementary algebra to $(\nu+a)^{2}<4 \nu G$, and the necessary and sufficient condition $T_{C}+T_{R} / \sqrt{r}<0$, written in terms of the parameters of the circuit, completes the statement.

Proposition 6. Assuming $\nu<G$, that is $R^{2} C<L$, and the additional hypotheses $b_{L}=a<\nu,(\nu+a)^{2}<4 \nu G$ and $\nu<b_{R}<G$, the circuit exhibits stable oscillations if and only if $\left(2 \nu-a-b_{R}\right) G<\nu^{2}-a b_{R}$.

These results complement the analysis done in [4], where the symmetric situation $b_{L}=b_{R}$ with $u=v=1$ was considered, and only the case $\ell, r<0$ was analyzed. 


\section{Preliminary Results}

First we prove the equivalence between systems (1) and (2).

The characteristic function $\phi$ induces a partition of $\mathbb{R}^{2}$ into three open strips separated by two straight lines, as follows

$$
\mathcal{S}_{L}=\{\mathbf{x}: \mathbf{x} \cdot \mathbf{k}<-u\}, \quad \mathcal{S}_{C}=\{\mathbf{x}:-u<\mathbf{x} \cdot \mathbf{k}<v\}, \quad \mathcal{S}_{R}=\{\mathbf{x}: \mathbf{x} \cdot \mathbf{k}>v\},
$$

and the straight lines are $\Gamma_{L}=\{\mathbf{x}: \mathbf{x} \cdot \mathbf{k}=-u\}$, and $\Gamma_{R}=\{\mathbf{x}: \mathbf{x} \cdot \mathbf{k}=v\}$. So system (1) is a piecewise linear differential system with three different linearity regions separated by two parallel straight lines. It can be written as

$$
\begin{array}{ll}
\dot{\mathbf{x}}=A \mathbf{x}+m_{L}(\mathbf{k} \cdot \mathbf{x}+u) \mathbf{b}-u \mathbf{b} & \text { in } \mathcal{S}_{L} \cup \Gamma_{L}, \\
\dot{\mathbf{x}}=B \mathbf{x} & \text { in } \Gamma_{L} \cup \mathcal{S}_{C} \cup \Gamma_{R}, \\
\dot{\mathbf{x}}=A \mathbf{x}+m_{R}(\mathbf{k} \cdot \mathbf{x}-v) \mathbf{b}+v \mathbf{b} & \text { in } \mathcal{S}_{R} \cup \Gamma_{R} .
\end{array}
$$

We note that systems (11) are analytic in $\mathbb{R}^{2} \backslash\left(\Gamma_{L} \cup \Gamma_{R}\right)$ but only of class $T_{C}^{0}$ on $\mathbb{R}^{2}$. Since they satisfy a Lipschitz condition in the whole $\mathbb{R}^{2}$, we can apply to systems (1) the classical theorems on existence, uniqueness and continuity on initial conditions and parameters. Note that their solutions are $C^{1}$, but not $C^{2}$.

The next result is proved in Proposition 4 of [27].

Lemma 7. System (1) having possible periodic orbits contained in two or three zones, can be written as

$$
\dot{x}=y-F(x), \quad \dot{y}=g(x),
$$

with $F(x)=t x+\phi(x)(T-t), \quad$ and $\quad g(x)=-d x-\phi(x)(D-d)$.

The next proposition shows that systems (1) and (2) are equivalent when $D>0$.

Proposition 8. System (1) with $D>0$, having possible periodic orbits contained in two or three zones, can be written as (2) where $F(x)$ and $g(x)$ are given by (3) and (4), respectively, with

$$
\begin{array}{cl}
T_{R} & =\frac{t+(T-t) m_{R}}{\sqrt{D}}, \quad T_{L}=\frac{t+(T-t) m_{L}}{\sqrt{D}}, \quad T_{C}=\frac{T}{\sqrt{D}}, \\
r & =\frac{d+(D-d) m_{R}}{D}, \quad \ell=\frac{d+(D-d) m_{L}}{D} .
\end{array}
$$

Proof. It follows from Lemma 7 that if system (1) has a periodic orbit then it can be written as system (2) with

$$
F(x)= \begin{cases}t x+(T-t)\left(m_{L}(x+u)-u\right) & \text { if } x \leq-u, \\ T x & \text { if }-u \leq x \leq v, \\ t x+(T-t)\left(m_{R}(x-v)+v\right) & \text { if } x \geq v\end{cases}
$$

and

$$
g(x)= \begin{cases}-d x-(D-d)\left(m_{L}(x+u)-u\right) & \text { if } x \leq-u \\ -D x & \text { if }-u \leq x \leq v \\ -d x-(D-d)\left(m_{R}(x-v)+v\right) & \text { if } x \geq v\end{cases}
$$


Equivalently we rewrite $F(x)$ and $g(x)$ in a more compact form as

$$
F(x)= \begin{cases}\tilde{T}_{L}(x+u)-T u & \text { if } x \leq-u \\ T x & \text { if }-u \leq x \leq v, \\ \tilde{T}_{R}(x-v)+T v & \text { if } x \geq v\end{cases}
$$

and

$$
g(x)= \begin{cases}-\tilde{\ell}(x+u)+D u & \text { if } x \leq-u \\ -D x & \text { if }-u \leq x \leq v \\ -\tilde{r}(x-v)-D v & \text { if } x \geq v\end{cases}
$$

where

$\tilde{T}_{R}=t+(T-t) m_{R}, \quad \tilde{T}_{L}=t+(T-t) m_{L}, \quad \tilde{r}=d+(D-d) m_{R}, \quad \tilde{\ell}=d+(D-d) m_{L}$.

Thus introducing the change of variables $(x, y, s) \mapsto(X, Y, \tau)$ given by

$$
X=x, \quad Y=\frac{y}{\sqrt{D}} \text { and } \tau=-\sqrt{D} s,
$$

we get that system (1) can be written as (2) satisfying (3) and (4) with

$$
T_{R}=\frac{\tilde{R}}{\sqrt{D}}, \quad T_{L}=\frac{\tilde{T}_{L}}{\sqrt{D}}, \quad T_{C}=\frac{T}{\sqrt{D}}, \quad r=\frac{\tilde{r}}{D}, \quad \ell=\frac{\tilde{\ell}}{D},
$$

and $(X, Y, \tau)$ instead of $(x, y, s)$. This completes the proof of the proposition.

Now we classify the equilibria of system (2).

Proposition 9. The following statement hold for the piecewise linear differential system (1) satisfying $D>0$ and written in the form (2).

(a) If $\ell \geq 0$ and $r \geq 0$, then the origin is the unique equilibrium, and it is hyperbolic.

(b) If $\ell \geq 0$ and $r<0$, then there are two equilibria, the origin and $e_{R}=$ $\left(x_{R}, y_{R}\right)=\left((r-1) v / r,\left(T_{C} r-T_{R}\right) v / r\right)$ in $\mathcal{S}_{R}$, the origin is hyperbolic and $e_{R}$ is a saddle.

(c) If $\ell<0$ and $r \geq 0$, then there are two equilibria: the origin and $e_{L}=$ $\left(x_{L}, y_{L}\right)=\left((1-\ell) u / \ell,\left(T_{L}-T_{C} \ell\right) u / \ell\right)$ in $\mathcal{S}_{L}$, the origin is hyperbolic and $e_{L}$ is a saddle.

(d) If $\ell<0$ and $r<0$, then there are three equilibria: the origin, $e_{L}$ in $S_{L}$ and $e_{R}$ in $S_{R}$, the origin is hyperbolic, and $e_{L}$ and $e_{R}$ are saddles.

Proof. The proof is straightforward, see for instance Theorem 2.15, or the hyperbolic singular point theorem of [7].

The next result will be useful when system (2) has more than one equilibrium point.

Lemma 10. Consider the Liénard differential system (2) with $r<0$ or $\ell<0$. If it has a periodic orbit then it is contained in the strip $x_{2}<x<x_{1}$ where

$$
x_{1}=\left\{\begin{array}{ll}
x_{R} & \text { if } r<0, \\
+\infty & \text { if } r \geq 0
\end{array} \text { and } x_{2}= \begin{cases}x_{L} & \text { if } \ell<0, \\
-\infty & \text { if } \ell \geq 0 .\end{cases}\right.
$$


Proof. We first prove the case $r<0$. Taking into account the behavior of the vector field on the line $x=x_{R}$, it follows that the periodic orbit is contained in the region $x<x_{R}$. Otherwise, since on the straight line $x=x_{R}$ we have $\dot{x}>0$ if $y>y_{R}$ and $\dot{x}<0$ if $y<y_{R}$, and the point $e_{R}$ of Proposition 9 is in the interior of the bounded region $V$ limited by the periodic orbit, we have a contradiction because the sum of the indices of the equilibrium points contained in $V$ is not equal to 1 , see for instance page 148 of [36].

The proof for the case $\ell<0$ follows in an analogous way.

We note that system (2) is invariant under the following symmetry:

$$
\left(x, y, t, T_{L}, T_{R}, \ell, r, u, v\right) \mapsto\left(-x,-y, t, T_{R}, T_{L}, r, \ell, v, u\right) .
$$

This symmetry will be useful to split the analysis of the system with three zones into different subcases with only two zones.

It is easy to check that the traces in the regions $\mathcal{S}_{L}, \mathcal{S}_{C}$ and $\mathcal{S}_{R}$ are given by $T_{L}$, $T_{C}$ and $T_{R}$, respectively. By the Bendixon theorem, see for instance Theorem 7.10 in [7], these traces cannot have the same sign when limit cycles exist.

Without loss of generality we can also assume that $T_{C}>0$. Clearly we can change the sign of $T_{C}$ doing the change of variables $(x, y, \tau) \mapsto(x,-y,-\tau)$. From now on in the rest of the paper we will assume that $T_{C}>0$. We remark that following the proofs it becomes clear that when $T_{C}<0$ the stability of the limit cycle (if exists) is unstable, because when $T_{C}>0$ it is stable.

We say that a vector field has the non-negative rotation property whenever along any half-ray starting from the origin the angle of the vector field measured with respect the positive direction of the $x$-axis does not decrease as long as one moves far from the origin.

We will use the Massera's method for uniqueness of limit cycles. To this end, we recall that a period annulus is a region in the plane completely filled by nonisolated periodic orbits. For a periodic orbit surrounding the origin, we say that it is star-like with respect to the origin when any segment joining the origin and a point of the periodic orbit has no other points in common with the periodic orbit, and consequently such segments are in the interior of the periodic orbit.

Theorem 11 (Massera's method). Consider a Liénard differential system $x^{\prime}=$ $F(x)-y$ and $y^{\prime}=g(x)$ defined in the open strip

$$
S=\left\{(x, y) \in \mathbb{R}^{2}: x_{2}<x<x_{1}\right\}
$$

for some $x_{2}<0<x_{1}$. Assume that $x g(x)>0$ for $x \in S$, and that $F(0)=0$, so that the only equilibrium point in $S$ is the origin. Assume also that the system in $S$ has the non-negative rotation property and has no period annulus surrounding the origin. If the system has a periodic orbit then it is star-like with respect to the origin and it is a limit cycle which is unique and stable.

Proof. Assume first that $x_{1}=\infty, x_{2}=-\infty$, i.e. system $x^{\prime}=F(x)-y$ and $y^{\prime}=g(x)$ has the origin as the unique equilibrium point. Then Theorem 11 is just Lemma 1 of $[25]$.

Now assume either $x_{1}=x_{R}$ or $x_{2}=x_{L}$. It follows from Lemma 10 that any periodic orbit must be contained in the strip $x_{2}<x<x_{1}$. Now we can apply the arguments of the proof of Lemma 1 of [25] to the strip $S$ and the theorem follows. 


\section{Computation of the points $Y_{ \pm}^{r}$ And $Y_{ \pm}^{\ell}$}

In this section we consider the system with two linearity zones, i.e., with either $T_{L}=T_{C}$ and $\ell=1$ (obtained by suppressing the left zone and extending the central zone to the left), or $T_{R}=T_{C}$ and $r=1$ (obtained by suppressing the right zone and extending the central zone to the right). Without loss of generality we can consider the case $T_{L}=T_{C}$ and $\ell=1$, because the other case can be studied in a similar way. Hence in this section we will work with the system

$$
x^{\prime}=F(x)-y, \quad \text { and } \quad y^{\prime}=g(x),
$$

where

$$
F(x)= \begin{cases}T_{C} x & \text { if } x<v \\ T_{R}(x-v)+T_{C} v & \text { if } x \geq v\end{cases}
$$

and

$$
g(x)= \begin{cases}x & \text { if } x<v \\ r(x-v)+v & \text { if } x \geq v\end{cases}
$$

Moreover we will consider the focus-saddle case, i.e. the case in which $T_{C} \in(-2,2)$ and $r<0$. To alleviate expressions, we can do a homogeneous rescaling by a factor of $v$, which is equivalent to assume $v=1$. At the end, it will suffice to multiply both coordinates by $v$ to undo the rescaling.

We suppose for the focus at the origin the eigenvalues $\sigma \pm i \omega$, where $\sigma^{2}+\omega^{2}=1$, and define the ratio $\gamma$ between the real and imaginary parts of such eigenvalues, so that

$$
2 \sigma=T_{C}, \quad \omega=\sqrt{1-\sigma^{2}}, \quad \gamma=\frac{\sigma}{\omega}=\frac{T_{C}}{\sqrt{4-T_{C}^{2}}} .
$$

With this notation, if $\left(x_{i}, y_{i}\right)$ is the starting point for an orbit using the focus dynamics and we want to know the final point $\left(x_{f}, y_{f}\right)$ along the orbit after a time $t$, then we can write the computations in terms of the phase angle $\theta=\omega t$ as follows,

$$
\left(\begin{array}{l}
x_{f} \\
y_{f}
\end{array}\right)=\exp (\gamma \theta)\left(\begin{array}{cc}
\cos \theta+\gamma \sin \theta & -\frac{\sin \theta}{\omega} \\
\frac{\sin \theta}{\omega} & \cos \theta-\gamma \sin \theta
\end{array}\right)\left(\begin{array}{l}
x_{i} \\
y_{i}
\end{array}\right) .
$$

We remark that the phase angle $\theta$ does not coincide in general with the geometrical angle of the orbit as seen from the focus at the origin, with the exception of the cases when $\theta=n \pi$ with $n \in \mathbb{Z}$.

Regarding the saddle point, their eigenvalues are denoted by $\lambda_{-}<0<\lambda_{+}$where

$$
\lambda_{ \pm}=\frac{T_{R} \pm \sqrt{T_{R}^{2}-4 r}}{2}
$$

so that

$$
T_{R}=\lambda_{+}+\lambda_{-}, \quad r=\lambda_{+} \cdot \lambda_{-},
$$

and we also introduce for convenience the notation

$$
\mu_{ \pm}=\frac{\lambda_{ \pm}}{r}=\frac{1}{\lambda_{\mp}}=\frac{T_{R} \pm \sqrt{T_{R}^{2}-4 r}}{2 r} .
$$


Note that the saddle point, originally at $\left(x_{R}, y_{R}\right)=\left(v-v / r, T_{C} v-T_{R} v / r\right)$, after the introduced rescaling, becomes the point $\left(1-1 / r, T_{C}-T_{R} / r\right)=\left(1-\mu_{+} \mu_{-}, 2 \sigma-\right.$ $\left.\mu_{+}-\mu_{-}\right)$. The $\lambda_{ \pm}$-eigenvectors of the matrix

$$
\left(\begin{array}{cc}
T_{R} & -1 \\
r & 0
\end{array}\right)=\left(\begin{array}{cc}
\lambda_{+}+\lambda_{-} & -1 \\
\lambda_{+} \lambda_{-} & 0
\end{array}\right)
$$

are $\left(1, \lambda_{\mp}\right)^{T}$. The linear $\lambda_{ \pm}$-invariant manifolds that emanate from the saddle following the eigenvectors $\left(1, \lambda_{\mp}\right)^{T}$ intersect the line $x=1$ at the points $\left(1, y_{ \pm}\right)$ when

$$
\left(\begin{array}{c}
1-\mu_{+} \mu_{-} \\
2 \sigma-\mu_{+}-\mu_{-}
\end{array}\right)+\alpha\left(\begin{array}{c}
1 \\
\lambda_{\mp}
\end{array}\right)=\left(\begin{array}{c}
1 \\
y_{ \pm}
\end{array}\right)
$$

that is, for $\alpha=\mu_{+} \mu_{-}=1 / r$. Thus

$$
y_{ \pm}=2 \sigma-\mu_{+}-\mu_{-}+\mu_{+} \mu_{-} \lambda_{\mp}=2 \sigma-\mu_{+}-\mu_{-}+\mu_{\mp}=2 \sigma-\mu_{ \pm} .
$$

To compute now the values $Y_{ \pm}^{r}$ it suffices to solve the equation

$$
\left(\begin{array}{c}
0 \\
Y_{ \pm}^{r}
\end{array}\right)=v \exp (\gamma \theta)\left(\begin{array}{cc}
\cos \theta+\gamma \sin \theta & -\frac{\sin \theta}{\omega} \\
\frac{\sin \theta}{\omega} & \cos \theta-\gamma \sin \theta
\end{array}\right)\left(\begin{array}{c}
1 \\
2 \sigma-\mu_{ \pm}
\end{array}\right),
$$

where we have added the factor $v$ to undo the rescaling, and the phase angle must satisfy $0< \pm \theta<\pi$, since we must integrate forward (backward) in time to get $Y_{+}^{r}$ $\left(Y_{-}^{r}\right)$, see Figure 2 .

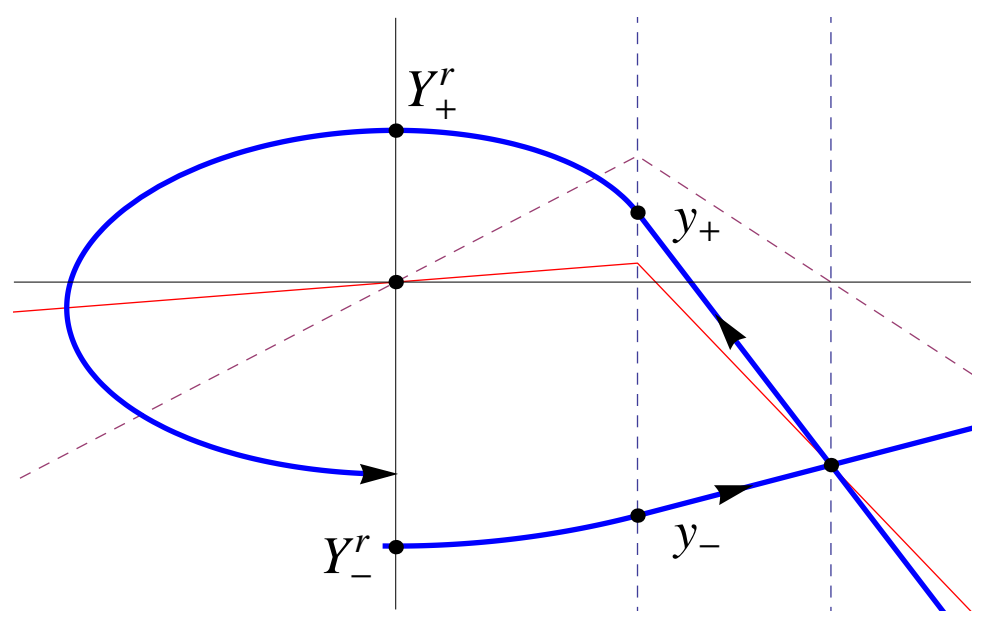

FiguRE 2. Remarkable points for $r<0,0<T_{C}<2$ and $T_{R}<0$. The graphs of functions $F(x)$ and $g(x)$ are drawn in solid and dashed, respectively. The vertical lines $x=v$ and $x=x_{R}$ are also drawn.

Assume first we want to compute $Y_{+}^{r}$. From the first coordinate, we get

$$
\cos \theta+\gamma \sin \theta-\left(2 \sigma-\mu_{+}\right) \frac{\sin \theta}{\omega}=\cos \theta-\gamma \sin \theta+\mu_{+} \frac{\sin \theta}{\omega}=0 .
$$

Equivalently, we write

$$
\omega \cos \theta=\left(\sigma-\mu_{+}\right) \sin \theta,
$$


and so, from

we obtain

$$
\tan \theta=\frac{\omega}{\sigma-\mu_{+}}
$$

$$
\cos \theta=\operatorname{sign}\left(\sigma-\mu_{+}\right) \frac{1}{\sqrt{1+\tan ^{2} \theta}}=\frac{\sigma-\mu_{+}}{\sqrt{\left(\sigma-\mu_{+}\right)^{2}+\omega^{2}}}=\frac{\sigma-\mu_{+}}{\sqrt{1-2 \sigma \mu_{+}+\mu_{+}^{2}}}
$$

and then

$$
\sin \theta=\frac{\omega}{\sqrt{1-2 \sigma \mu_{+}+\mu_{+}^{2}}}
$$

To avoid problems with the determination of the arctan function, since it is not difficult to build examples with $\theta>\pi / 2$, we use the trigonometric identity

$$
\tan \frac{\theta}{2}=\frac{\sin \theta}{1+\cos \theta}
$$

leading to

$$
\theta=2 \arctan \left(\frac{\omega}{\sqrt{1-2 \sigma \mu_{+}+\mu_{+}^{2}}+\sigma-\mu_{+}}\right) .
$$

Taking into account that, from (16) we know that

$$
\cos \theta-\gamma \sin \theta=-\mu_{+} \frac{\sin \theta}{\omega}
$$

we finally obtain from the second coordinate of (15) that

$$
Y_{+}^{r}=v \exp (\gamma \theta) \frac{1-\mu_{+}\left(2 \sigma-\mu_{+}\right)}{\sqrt{1-2 \sigma \mu_{+}+\mu_{+}^{2}}}=v \exp (\gamma \theta) \sqrt{1-2 \sigma \mu_{+}+\mu_{+}^{2}},
$$

where $\theta$ is given in (17).

The computations for $Y_{-}^{r}$ are identical if we change $\theta$ by $-\theta$ and $\mu_{+}$by $\mu_{-}$. To eliminate any ambiguity however, and to use also a positive angle $\theta$ in $(0, \pi)$, we start from

$$
\left(\begin{array}{c}
0 \\
Y_{-}^{r}
\end{array}\right)=v \exp (-\gamma \theta)\left(\begin{array}{cc}
\cos \theta-\gamma \sin \theta & \frac{\sin \theta}{\omega} \\
-\frac{\sin \theta}{\omega} & \cos \theta+\gamma \sin \theta
\end{array}\right)\left(\begin{array}{c}
1 \\
2 \sigma-\mu_{-}
\end{array}\right)
$$

solving for

leading now to

$$
\cos \theta-\gamma \sin \theta+\frac{\sin \theta}{\omega}\left(2 \sigma-\mu_{-}\right)=0
$$

$$
\omega \cos \theta=-\left(\sigma-\mu_{-}\right) \sin \theta, \quad \tan \theta=-\frac{\omega}{\sigma-\mu_{-}},
$$

so that

$$
\cos \theta=-\frac{\sigma-\mu_{-}}{\sqrt{1-2 \sigma \mu_{-}+\mu_{-}^{2}}}
$$

and

$$
\sin \theta=\frac{\omega}{\sqrt{1-2 \sigma \mu_{-}+\mu_{-}^{2}}}
$$


Using now that

we see that

$$
\cos \theta+\gamma \sin \theta=\mu_{-} \frac{\sin \theta}{\omega}
$$

$$
Y_{-}^{r}=-v \exp (-\gamma \theta) \frac{1-\mu_{-}\left(2 \sigma-\mu_{-}\right)}{\sqrt{1-2 \sigma \mu_{-}+\mu_{-}^{2}}}=-v \exp (-\gamma \theta) \sqrt{1-2 \sigma \mu_{-}+\mu_{-}^{2}},
$$

where $\theta$ is given by

$$
\theta=2 \arctan \left(\frac{\omega}{\sqrt{1-2 \sigma \mu_{-}+\mu_{-}^{2}}-\left(\sigma-\mu_{-}\right)}\right)
$$

Therefore the common expression for both ordinates is

$$
Y_{ \pm}^{r}= \pm v \exp \left( \pm \gamma \theta_{ \pm}\right) \sqrt{1-2 \sigma \mu_{ \pm}+\mu_{ \pm}^{2}}
$$

with

$$
\theta_{ \pm}=2 \arctan \left(\frac{\omega}{\sqrt{1-2 \sigma \mu_{ \pm}+\mu_{ \pm}^{2}} \pm\left(\sigma-\mu_{ \pm}\right)}\right)
$$

In a similar way we obtain $Y_{ \pm}^{\ell}$.

\section{Proof of Theorem 1}

In this section we consider system (13). Then Theorem 1 can be stated as follows.

Theorem 12. Consider the differential system (13) with $T_{C} \neq 0$. Then the following statements hold.

(a) Two necessary conditions for the existence of periodic orbits are $\left|T_{C}\right|<2$ and $T_{R} T_{C}<0$.

(b) If $\left|T_{C}\right|<2$ and $T_{R} T_{C}<0$ then the system has periodic orbits

(b.1) when $T_{C}>0$ and $r>0$ if and only if $T_{C}+T_{R} / \sqrt{r}<0$;

(b.2) when $T_{C}<0$ and $r>0$ if and only if $T_{C}+T_{R} / \sqrt{r}>0$;

(b.3) when $T_{C}>0$ and $r<0$ if and only if $e^{\pi T_{C} / \sqrt{4-T_{C}^{2}}} Y_{+}^{r}+Y_{-}^{r}<0$;

(b.4) when $T_{C}<0$ and $r<0$ if and only if $e^{\pi T_{C} / \sqrt{4-T_{C}^{2}}} Y_{+}^{r}+Y_{-}^{r}>0$.

In all cases the origin is surrounded by a limit cycle which is unique, stable if $T_{C}>0$ and unstable if $T_{C}<0$.

Now we shall prove Theorem 12 when $T_{C}>0$. The case $T_{C}<0$ can be proved in a similar way. Note that the traces in the regions $\mathcal{S}_{C}, \mathcal{S}_{R}$ are given by $T_{C}$ and $T_{R}$, respectively. By the Bendixon theorem, see for instance Theorem 7.10 in [7], they cannot have the same sign for the existence of limit cycles and thus $T_{R} T_{C}<0$. First we show that system (13) can be transformed in another system with the non-negative rotation property. This is the statement of the following proposition, where we prove more things that we will use later on.

Proposition 13. We restrict our attention to system (13) with $T_{C}>0$ in the region $x<x_{R}$ when $r<0$. We assume that $T_{R} \leq T_{C}$. Then the system can be transformed in another system having the non-negative rotation property if $T_{R}-$ $T_{C} r \leq 0$, or if $T_{R}-T_{C} r>0, r<0$ and $T_{R} \leq 0$. 
Proof. To show the non-negative rotation property, we will compute the slope of the vector field along half-rays of the form $y=\lambda x$. Since it will appear $F(x)-\lambda x$ in some denominators we can disregard the points of vertical slope in which $F(x)=\lambda x$.

Now we transform the system by introducing a new first variable $z=z(x)$, namely

$$
z=\operatorname{sgn}(x) \sqrt{2 G(x)} \text { where } G(x)=\int_{0}^{x} g(s) d s .
$$

Note that $z=x$ for $x \leq v$ and that this change of variable is injective for $x>v$ as long as $g(x)>0$, that is, for all $x$ when $r \geq 0$, or for $x<x_{R}$ when $r<0$. Note that $z^{2}(x)=2 G(x)$ and thus we have $z(x) z^{\prime}(x)=g(x)$ for all $x$, and so

$$
\frac{d z}{d x}=\frac{g(x)}{z} .
$$

Therefore

and in the new variables the system is

$$
\frac{d z}{d t}=\frac{d z}{d x} \frac{d x}{d t}=\frac{g(x)}{z}(F(x)-y),
$$

$$
\frac{d y}{d z}=\frac{d y / d t}{d z / d t}=\frac{z}{F(x(z))-y}
$$

which is equivalent to the system $\dot{z}=F(x(z))-y, \dot{y}=z$, where the dot now indicates the derivative with respect to an implicitly defined, different time parameterization of the orbits. Now we study the slope of the vector field along the half-rays of the form $y=\lambda z$.

Then we can write

$$
m_{\lambda}(z)=\left.\frac{d y}{d z}\right|_{y=\lambda z}=\frac{z}{F(x(z))-\lambda z},
$$

and to analyze the monotone character of this slope along the half-rays we compute its derivative with respect to $z$, namely

$$
\frac{d m_{\lambda}(z)}{d z}=\frac{\left.F(x(z))-\lambda z-z\left[F^{\prime}(x(z)) x^{\prime}(z)\right)-\lambda\right]}{[F(x(z))-\lambda z]^{2}},
$$

not to be considered at $z=v$, where this derivative has a jump discontinuity.

In the region $x<v$, since $z=x$ we have

$$
\frac{d m_{\lambda}(z)}{d z}=\frac{F(x(z))-z F^{\prime}(x(z)) x^{\prime}(z)}{[F(x(z))-\lambda z]^{2}}=\frac{T_{C} z-T_{C} z}{[F(x(z))-\lambda z]^{2}}=0 .
$$

So the slope of the vector field is constant along the half-rays.

For the region $x>v$ we get

$$
\frac{d m_{\lambda}(z)}{d z}=\frac{\left(T_{C}-T_{R}\right) v-T_{R}\left(z x^{\prime}(z)-x\right)}{[F(x(z))-\lambda z]^{2}} .
$$

Now we study the value of $z x^{\prime}(z)-x(z)$ for $x>v$. From (21) and the equality $z^{2}=2 G(x)=r(x-v)^{2}+2 v x-v^{2}$ for $x>v$, we have

$$
\begin{aligned}
z x^{\prime}(z)-x(z) & =\frac{z^{2}-x g(x)}{g(x)}=\frac{r(x-v)^{2}+2 v x-v^{2}-r x(x-v)-v x}{g(x)} \\
& =\frac{(x-v)(r x-r v+v-r x)}{g(x)}=\frac{v(1-r)(x-v)}{g(x)} .
\end{aligned}
$$


Then (22) can be rewritten as

$$
\frac{d m_{\lambda}(z)}{d z}=v \frac{\left(T_{C}-T_{R}\right) g(x)-T_{R}(1-r)(x-v)}{g(x)[F(x(z))-\lambda z]^{2}},
$$

and the sign of the above expression, as long as $g(x)>0$, is controlled by the sign of the numerator, namely

$\left(T_{C}-T_{R}\right) v+\left[\left(T_{C}-T_{R}\right) r-T_{R}(1-r)\right](x-v)=\left(T_{C}-T_{R}\right) v-\left(T_{R}-T_{C} r\right)(x-v)$, an expression which is affine in $x-v$.

Since our hypothesis implies $T_{C}-T_{R} \geq 0$, we see that the first constant term is always non-negative. If $T_{R}-T_{C} r \leq 0$ then the second term is also non-negative for $x>v$ and we are done. The case $T_{R}-T_{C} r>0$ leads always to a change in the sign of the expression for $z$ big enough, so that we cannot guarantee the monotone increasing character of the slope along half-rays in the whole plane. When $r<0$ however, we only need to study what happens for $x<x_{R}$, that is for $x-v<-v / r$. Substituting now $x=x_{R}$ in the above expression, we get

$$
\left(T_{C}-T_{R}\right) v+\left(T_{R}-T_{C} r\right) \frac{v}{r}=-T_{R} v+T_{R} \frac{v}{r}=-T_{R} v\left(1-\frac{1}{r}\right),
$$

which for $T_{R} \leq 0$ is non-negative. Thus we can also assure in such a case the required monotonicity for $x<x_{R}$, and the proposition follows.

Noticing that for $T_{R} \leq 0$ the hypothesis $T_{C}-T_{R} \geq 0$ is strictly satisfied, we see immediately that we can have $T_{R}-T_{C} r \leq 0$ (and then Proposition 13 applies) or $T_{R}-T_{C} r>0$, that is $r<T_{R} / T_{C} \leq 0$, but then since $T_{R} \leq 0$ Proposition 13 also applies. In short we get the following result.

Corollary 14. We restrict our attention to system (13) with $T_{C}>0$ in the region $x<x_{R}$ when $r<0$. If $T_{R} \leq 0$ then the system can be transformed in another system having the non-negative rotation property independently on the value of $r$.

Now we show that if system (13) has a periodic orbit, then it is unique.

Proposition 15. Assume that system (13) with $T_{C}>0$ has a periodic orbit. Then the periodic orbit surrounds the origin and it is a limit cycle which is unique and stable.

Proof. By Proposition 13 the system can be transformed into one which has the non-negative rotation property. Therefore, by Theorem 11 such a periodic orbit is a limit cycle which is unique. Applying Theorem 11 we get that the limit cycle of system (13) is stable because we are assuming that $T_{C}>0$.

To prove statement (b) we need to study the existence of periodic orbits surrounding the origin. To do this, we will use the positive and negative parts of the $y$-axis as domain and range for defining two different half-return maps, namely a right half-return map $P_{R}$ and a left half-return map $P_{L}$.

We start by studying the left half-return map $P_{L}$ defined in the positive $y$-axis, by taking the orbit starting at the point $(0, y)$ with $y>0$, and coming back to the negative $y$-axis at the point $\left(0,-P_{L}(y)\right)$. Since the system becomes purely linear, it is easy to see, see for instance [13] that $P_{L}$ is a linear function given by $P_{L}(y)=e^{\pi T_{C} / \sqrt{4-T_{C}^{2}}} y$. 
Now we study the qualitative properties of the right half-return map $P_{R}$ defined in the negative $y$-axis, by taking the orbit starting at the point $(0,-y)$ with $y>0$, and coming back to the positive $y$-axis at the point $\left(0, P_{R}(y)\right)$. The following lemma is Lemma 3 of [25].

Lemma 16. Consider a Liénard differential system with a continuous vector field given by $\dot{x}=F(x)-y$ and $\dot{y}=g(x)$. Assume that $F(x)$ is positive and increasing for small positive values of $x$, it has a positive zero only at $x=\bar{x}>0$, and it is decreasing to $-\infty$ as $x \rightarrow \infty$ monotonically for $x>\bar{x}$. Assuming also that $g(0)=0$ and $g(x)>0$ for all $x>0$, the following statements hold. The orbits starting at the point $(0,-y)$ with $y>0$, enter the half-plane $x>0$ and go around the origin in a counterclockwise path, coming back to the $y$-axis at the point $\left(0, P_{R}(y)\right)$ with $\left.P_{R}(y)\right)>0$. The difference $P_{R}(y)-y$ is positive for small values of $y$, but eventually becomes negative and tends to $-\infty$ when $y \rightarrow \infty$.

The map $P_{R}$ when $r<0$ is not defined for all positive values of $y$. We shall compute now its domain of definition. Two separatrices of saddle $\left(x_{R}, y_{R}\right)$ intersect the line $x=v$, the stable at the point $\left(v, y_{-}\right)$and the unstable at the point $\left(v, y_{+}\right)$, where

$$
y_{ \pm}=\frac{\left(2 T_{C} r-T_{R} \mp \sqrt{T_{R}^{2}-4 r}\right) v}{2 r},
$$

see Section 4 and Figure 2. The flow of system (13) in $x<v$ starting at the point $\left(v, y_{-}\right)$in backward time intersects the negative $y$-axis at the point $\left(0, Y_{-}^{r}\right)$.

The flow of system (13) in $x<v$ starting at the point $\left(v, y_{+}\right)$in forward time intersects the positive $y$-axis at the point $\left(0, P_{R}\left(-Y_{-}^{r}\right)\right)$, where it is assumed

$$
P_{R}\left(-Y_{-}^{r}\right)=\lim _{y \nearrow\left(-Y_{-}^{r}\right)} P_{R}(y)=Y_{+}^{r} .
$$

Hence the $\operatorname{map} P_{R}:\left(0,-Y_{-}^{r}\right) \rightarrow\left(0, Y_{+}^{r}\right)$.

Corollary 17. Assume that system (13) has $T_{C}>0$. Then the orbits starting at the point $(0,-y)$ with $y>0$, enter the half-plane $x>0$ and go around the origin in a counterclockwise path, coming back to the $y$-axis at the point $\left(0, P_{R}(y)\right)$ with $\left.P_{R}(y)\right)>0$. The difference $P_{R}(y)-y$ is positive for small values of $y$, and

(a) it eventually becomes negative and tends to $-\infty$ when $y \rightarrow \infty$, if $r>0$;

(b) tends to $Y_{+}^{r}+Y_{-}^{r}$ when $y \rightarrow-Y_{-}^{r}$, if $r<0$.

Proof. Since we are assuming that $T_{C}>0$ and $T_{R}<0$ we get that $F(x)$ is positive and increasing for small positive values of $x$, and it has a positive zero only at $x=v\left(1-T_{C} / T_{R}\right)>0$. It is decreasing to $-\infty$ as $x \rightarrow \infty$ monotonically for $x>v\left(1-T_{C} / T_{R}\right)$. Note that $g(0)=0$ and, when $r>0, g(x)>0$ for all $x>0$. Now we are under the assumptions of Lemma 16 and the statement (a) of the corollary follows from it.

The proof of statement (b) follows directly from the domain of definition of the map $P_{R}$ and its image.

Now we continue with the proof of Theorem 12 and we need to show that in fact, we have a periodic orbit. Note that for system (13) the origin is a node when $\left|T_{C}\right| \geq 2$, and a focus when $\left|T_{C}\right|<2$. Moreover, it cannot be a node because some of its invariant manifolds are straight lines that should extend to the infinity, 
precluding the existence of periodic orbits. Then it must be a focus and thus $\left|T_{C}\right|<2$. This concludes the proof of statement (a).

Now we prove statements (b.1) and (b.3). Statement (b.1) can be proved with exactly the same proof as in the proof of Theorem 2 in [25]. In this case, i.e. when $r>0$ we can show that a necessary and sufficient condition for the existence of periodic orbits in this case is that

$$
T_{C}+\frac{T_{R}}{\sqrt{r}}<0
$$

Now we prove statement (b.3), i.e. we need to study the existence of periodic orbits when $r<0$. Note that as explained above we have that

$$
P_{L}(y)=e^{\pi T_{C} / \sqrt{4-T_{C}^{2}}} y
$$

and in particular

$$
P_{L}\left(Y_{+}^{r}\right)=e^{\pi T_{C} / \sqrt{4-T_{C}^{2}}} Y_{+}^{r} .
$$

If $P_{L}\left(Y_{+}^{r}\right)<-Y_{-}^{r}$, as shown in Figure 2, then it is easy to conclude the existence of a trapping region that, since the focus at the origin is unstable, assures the existence of a periodic orbit. Its uniqueness and stability comes from Proposition 15.

Suppose that $P_{L}\left(Y_{+}^{r}\right)=-Y_{-}^{r}$. Then we have a homoclinic connection. We claim that inside the region limited by the homoclinic loop there are no periodic solutions. For proving the claim we shall use the next result, see Theorem 1 in page 364 of [31].

Theorem 18. Let $p$ be a topological saddle of an analytic differential system in the plane and let $\gamma$ be a homoclinic loop at $p$. Then the orbits near $\gamma$ contained in the region limited by $\gamma$ tend to $\gamma$ in forward (respectively backward) time if and only the trace of the linear part of the system at $p$ is negative (respectively positive).

Corollary 19. Let $e_{R}$ be a saddle of the Liénard piecewise linear differential system (2) and assume that this saddle has a homoclinic loop $\gamma$. Then the orbits near $\gamma$ contained in the region limited by $\gamma$ tend to $\gamma$ in forward (respectively backward) time if and only the trace of the linear part of the system at $p$ is negative (respectively positive).

Proof. Since the Liénard piecewise linear differential system (2) can be a limit of analytic differential systems in the plane having homoclinic loops tending to the homoclinic loop $\gamma$ of system (2), by Theorem 18 the corollary follows.

Now we prove the claim. Since $T_{R}<0$ the trace of the saddle $e_{R}$ is negative. By Corollary 19 the homoclinic loop surrounding the focus is stable. By Proposition 15 inside the region limited by the loop there is at most one periodic solution surrounding the focus and it is stable. But this is in contradiction with the fact that the homoclinic loop is stable. Hence the claim is proved.

If finally $P_{L}\left(Y_{+}^{r}\right)>-Y_{-}^{r}$ then we have a trapping region in backward time. By considering that the focus is stable when the time is reversed, the only possibilities for periodic orbits are a semi-stable periodic orbit, or two or more periodic orbits, again in contradiction with Proposition 15. Statement (b.3) is shown and this concludes the proof of Theorem 12 . 


\section{Proof of Theorem 3}

First we recall one of the main results of [25] which we shall use for proving Theorem 3.

In [25] the authors studied the limit cycles of the Liénard piecewise linear differential systems of the form

$$
\dot{x}=F(x)-y, \quad \dot{y}=g(x)-\delta,
$$

where

and

$$
F(x)= \begin{cases}t_{L}(x+1)-t_{C}, & \text { if } x \leq-1 \\ t_{C} x, & \text { if }|x| \leq 1 \\ t_{R}(x-1)+t_{C}, & \text { if } x \geq 1\end{cases}
$$

$$
g(x)= \begin{cases}d_{L}(x+1)-d_{C}, & \text { if } x \leq-1, \\ d_{C} x, & \text { if }|x| \leq 1, \\ d_{R}(x-1)+d_{C}, & \text { if } x \geq 1\end{cases}
$$

where $d_{C}>0,-d_{C}<\delta<d_{C}$ and $d_{L}, d_{R} \geq 0$. Theorem 1 of [25] states:

Theorem 20. Consider the differential system (26)-(27) with only one equilibrium point in the central zone (i.e. $\left.d_{C}>0,-d_{C}<\delta<d_{C}\right)$, and $d_{L}, d_{R} \geq 0$. If the external traces satisfy $t_{L}, t_{R}<0$, while the central trace is positive, that is $t_{C}>0$, then the equilibrium point is surrounded by a limit cycle which is unique and stable.

We consider the change of variables $(x, y) \mapsto(X, Y)$ given by

$$
X=x-\bar{x}, \quad Y=\frac{y-t_{C} \bar{x}}{\sqrt{d_{C}}}, \quad \tau=\sqrt{d_{C}} s,
$$

where $\bar{x}=\delta / d_{C}$. Then the differential system (26)-(27) becomes

$$
X^{\prime}=Y-F(X), \quad Y^{\prime}=g(X),
$$

where

and

$$
F(X)= \begin{cases}\frac{t_{L}}{\sqrt{d_{L}}}\left(X-X_{L}\right)-\frac{t_{C}}{\sqrt{d_{C}}} X_{L} & \text { if } X \leq X_{L}, \\ \frac{t_{C}}{\sqrt{d_{C}}} X & \text { if } X_{L} \leq X \leq X_{R} \\ \frac{t_{R}}{\sqrt{d_{C}}}\left(X-X_{R}\right)+\frac{t_{C}}{\sqrt{d_{C}}} X_{R} & \text { if } x \geq X_{R},\end{cases}
$$

$$
g(X)= \begin{cases}\frac{d_{L}}{d_{C}}\left(X-X_{L}\right)+X_{L} & \text { if } X \leq X_{L}, \\ X & \text { if } X_{L} \leq X \leq X_{R} \\ \frac{d_{R}}{d_{C}}\left(X-X_{R}\right)+X_{R} & \text { if } X \geq X_{R},\end{cases}
$$

where $X_{R}=1-\bar{x}, X_{L}=-1-\bar{x}$ and comparing with system (2)-(4) we have

$$
\begin{gathered}
T_{L}=\frac{t_{L}}{\sqrt{d_{C}}}, \quad T_{C}=\frac{t_{C}}{\sqrt{d_{C}}}, \quad T_{R}=\frac{t_{R}}{\sqrt{d_{C}}}, \\
r=\frac{d_{R}}{d_{C}} \geq 0, \quad \ell=\frac{d_{L}}{d_{C}} \geq 0, \quad X_{L}=-u, \quad X_{R}=v .
\end{gathered}
$$


We note that if the parameters of system (2)-(4) satisfies the conditions (28), then from Theorem 20 it follows statements (b) and (c) of Theorem 3. Since conditions (28) imply that

$$
r \geq 0, \quad \ell \geq 0 \text { and } \quad u+v=2,
$$

it follows that Theorem 20 does not cover all the cases of statements (b) and (c) of Theorem 3. In any case, Theorem 20 never provides information about statements (d) or (e) of Theorem 3.

In what follows we shall prove Theorem 3, using the same ideas of the proof of Theorem 20. Moreover in Theorem 20 it is only considered the case that the traces in the regions $S_{L}, S_{C}$ and $S_{R}$ have signs -, + and -, respectively.

To prove Theorem 3 we will state and prove several auxiliary results.

The next result proves the uniqueness of the limit cycle (if it exists) in Theorem 3 .

Proposition 21. Assume that system (2) with $T_{C}>0$ has three linearity zones and satisfies one of the following conditions.

(a) The sign of $T_{R}$ and $T_{L}$ are equal and different from the sign of $T_{C}$, that is, $T_{R}, T_{L} \leq 0$

(b) $T_{L} \leq 0$ but $T_{R}>0$ and we have $T_{R}-T_{C} r \leq 0$;

(c) $T_{R} \leq 0$ but $T_{L}>0$ and we have $T_{L}-T_{C} \ell \leq 0$.

If system (2) has a periodic orbit, then it surrounds the origin and it is a limit cycle which is unique and stable.

Proof. The proof of statement (c) is very similar to the proof of statement (b), so we do not do it. First we consider system (2) restricted to $x \geq 0$, that it can be considered with only two linearity zones as system (13) restricted to $x \geq 0$. We shall prove simultaneously statements (a) and (b).

From Proposition 13 and Corollary 14 system (13) can be transformed into another having the non-negative rotation property for all the half-rays contained in $x \geq 0$. By using the symmetry given in (12), and applying again Proposition 13 and Corollary 14 , we can deduce that such systems can also be transformed into an equivalent system having the non-negative rotation property for all the half-rays contained in $x \leq 0$. Note that the change of variables $(20)$ is the same in the whole plane, i.e. in $x \geq 0$ and in $x \leq 0$. In other words, the change of variables $(20)$ produce that simultaneously in $x \leq 0$ and $x \geq 0$ the non-negative rotation property holds. In short, system (2) with three linearity zones can be transformed into a system with the non-negative rotation property for all the half-rays contained in $S$. Consequently from Theorem 11 we conclude that for a such system, if there exists a periodic orbit, then it surrounds the origin and it is a limit cycle that is unique and stable.

To prove statement (b) of Theorem 3 we need to prove the existence of such a periodic orbit surrounding the origin. To do this, again we will use the positive and negative parts of the $y$-axis as domain and range for defining two different half-return maps, namely a right half-return map $P_{R}$ and a left half-return map $P_{L}$.

We start by studying the qualitative properties of the right half-return map $P_{R}$ defined in the negative $y$-axis, by taking the orbit starting at the point $(0,-y)$ with 
$y>0$, and coming back to the positive $y$-axis at the point $\left(0, P_{R}(y)\right)$. We have the following result whose proof is exactly the same as Corollary 17 .

Corollary 22. Assume that system (2) has three linearity zones, and that the signs of $T_{R}$ and $T_{L}$ are equal and different from the sign of $T_{C}>0$. Then the orbits starting at the point $(0,-y)$ with $y>0$, enter the half-plane $x>0$ and go around the origin in a counterclockwise path, coming back to the $y$-axis at the point $\left(0, P_{R}(y)\right)$ with $\left.P_{R}(y)\right)>0$. The difference $P_{R}(y)-y$ is positive for small values of $y$, eventually becomes negative and

(a) tends to $-\infty$ when $y \rightarrow \infty$ if $r>0$,

(b) tends to $Y_{+}^{r}+Y_{-}^{r}$ when y $\nearrow\left(-Y_{-}^{r}\right)$, if $r<0$.

Now we can do a similar study for the left half-return map $P_{L}$ defined in the positive $y$-axis, by taking the orbit starting at the point $(0, y)$, with $y>0$, and coming back to the negative $y$-axis at the point $\left(0,-P_{L}(y)\right)$. More precisely we have the following result.

Corollary 23. Assume that system (2) has three linearity zones, and that the signs of $T_{R}$ and $T_{L}$ are equal and different from the sign of $T_{C}>0$. Then the orbits starting at the point $(0, y)$, with $y>0$, enter the half-plane $x<0$ and go around the origin in a counterclockwise path, coming back to the $y$-axis at the point $\left(0,-P_{L}(y)\right)$ with $P_{L}(y)>0$. The difference $P_{L}(y)-y$ is positive for small values of $y$, eventually becomes negative and

(a) tends to $-\infty$ when $y \rightarrow \infty$, if $\ell>0$,

(b) tends to $Y_{+}^{\ell}+Y_{-}^{\ell}$ when $y \nearrow Y_{+}^{\ell}$, if $\ell<0$.

With these results we can prove Theorem 3 .

Proof of Theorem 3. Note that the traces in the regions $\mathcal{S}_{L}, \mathcal{S}_{C}$ and $\mathcal{S}_{R}$ are given by $T_{L}, T_{C}$ and $T_{R}$, respectively. By the Bendixon theorem, see for instance Theorem 7.10 in [7], they cannot have the same sign for the existence of limit cycles. This proves statement (a) of the theorem.

Statements (c), (d) and (e) are immediate consequences of Proposition 21.

To prove statement (b) we first look for the existence of periodic orbits by showing that system (2) has at least one periodic orbit. The existence of periodic orbits is equivalent to the existence of two positive values $y_{L}$ and $y_{R}$ such that

$$
P_{R}\left(y_{R}\right)=y_{L}, \quad y_{R}=P_{L}\left(y_{L}\right) .
$$

Adding and subtracting the above equations we get an equivalent system of sufficient and necessary conditions for the existence of periodic orbits, namely

$$
\begin{aligned}
& P_{R}\left(y_{R}\right)+y_{R}=y_{L}+P_{L}\left(y_{L}\right), \\
& P_{R}\left(y_{R}\right)-y_{R}=y_{L}-P_{L}\left(y_{L}\right) .
\end{aligned}
$$

Furthermore, conditions (29) for the existence of periodic orbits translate now to the existence of a value $Y>0$ being solution of the single equation $\widehat{P}_{R}(Y)=-\widehat{P}_{L}(Y)$, that is, of

$$
\widehat{P}_{R}(Y)+\widehat{P}_{L}(Y)=0,
$$

where

$$
\widehat{P}_{R}(Y)=P_{R}\left(y_{R}\right)-y_{R}, \quad \widehat{P}_{L}(Y)=P_{L}\left(y_{L}\right)-y_{L},
$$


being $y_{R}$ the unique solution of $Y=P_{R}\left(y_{R}\right)+y_{R}$ and $y_{L}$ the unique solution of $Y=P_{L}\left(y_{L}\right)+y_{L}$ (we recall that $P_{R}$ and $P_{L}$ are monotone functions).

The function $\widehat{P}_{R}(Y)+\widehat{P}_{L}(Y)$ is positive for small values $Y>0$ and, when $r>0$ and $\ell>0$, such function is negative when $Y \rightarrow \infty$ as a consequence of statement (a) of Corollaries 22 and 23.

Now we apply the mean value theorem to conclude the existence of at least a solution, and so a periodic orbit of the system. The uniqueness and the stability of the periodic orbit come from Proposition 21. This concludes the proof of statement (b).

\section{ACKNOWLEDGEMENTS}

The first author is partially supported by a MINECO/FEDER grant MTM200803437, a CIRIT grant number 2009SGR-410, an ICREA Academia, two grants FP7-PEOPLE-2012-IRSES 316338 and 318999, and FEDER-UNAB10-4E-378.

The second author was supported by MINECO/FEDER grant MTM2012-31821.

The third author was supported by Portuguese National Funds through FCT Fundação para a Ciência e a Tecnologia within the project PTDC/MAT/117106/2010 and by CAMGSD.

\section{REFERENCES}

[1] T. Carletti and G. Villari, A note on existence and uniqueness of limit cycles for Liénard systems, J. Math. Anal. Appl. 307 (2005), 763-773.

[2] V. Carmona, E. Freire, E. Ponce and F. Torres, On simplifying and classifying piecewise linear systems, IEEE Trans. Circuits and Systems I: Fundamental Theory and Applications 49 (2002), 609-620.

[3] L.O. ChuA, Memristor: The missing circuit element, IEEE Trans. Ciruit Theory, vol. CT18, 507-519

[4] F. Corinto, A. Ascoli And M. Gilli, Nonlinear Dynamics of Memristor Oscillators, IEEE Trans. Ciruits and Systems I: Regular papers, vol. 58, 1323-1336.

[5] M. di Bernardo, C. J. Budd, A. R. Champneys and P. Kowalczyk, Piecewise-smooth Dynamical Systems: Theory and Applications, Springer Verlag, London, 2008.

[6] F. DUMORTIER AND C. LI, On the uniqueness of limit cycles surrounding one or more singularities for Liénard equations, Nonlinearity 9 (1996), 1489-1500.

[7] F. Dumortier, J. Llibre And J.C. ArTÉs, Qualitative theory of planar differential systems, Universitext, Springer-Verlag, Berlin, 2006.

[8] F. Dumortier And C. Rousseau, Cubic Liénard equations with linear damping, Nonlinearity 3 (1990), 1015-1039.

[9] E. Freire, E. Ponce, F. Rodrigo And F. Torres, Bifurcation sets of continuous piecewise linear systems with two zones, Int. J. Bifurcations and Chaos 8 (1998), 2073-2097.

[10] E. Freire, E. Ponce, F. Rodrigo And F. Torres, Bifurcation sets of continuous piecewise linear systems with three zones, Int. J. Bifurcations and Chaos 12 (2002), 1675-1702.

[11] E. Freire, E. PONCE AND J. Ros, Limit cycle bifurcation from center in symmetric piecewise-linear systems, Int. J. Bifurcation and Chaos 9 (1999), 895-907.

[12] E. Freire, E. Ponce And F. TORREs, Hopf-like bifurcations in planar piecewise linear systems, Publicacions Matemàtiques 41 (1997), 135-148.

[13] E. Freire, E. Ponce And F. Torres, Canonical discontinuous planar piecewise linear systems, SIAM J. Appl. Dyn. Sys., 11 (2012), 181-211.

[14] A. Gasull, H. Giacomini and J. Llibre, New criteria for the existence and non-existence of limit cycles in Liénard differential systems, Dynamical Systems: An International Journal 24 (2009), 171-185.

[15] F. Giannakopoulos And K. Pliete, Planar systems of piecewise linear differential equations with a line of discontinuity, Nonlinearity 14 (2001), 1611-1632. 
[16] D. Hilbert, Mathematische Probleme, Lecture, Second Internat. Congr. Math. (Paris, 1900), Nachr. Ges. Wiss. G"ttingen Math. Phys. KL. (1900), 253-297; English transl., Bull. Amer. Math. Soc. 8 (1902), 437-479.

[17] S. J. Hogan, Relaxation oscillations in a system with a piecewise smooth drag coefficiente, J. Sound and Vibration 263 (2003), 467-471.

[18] W. T. VAN Horssen, On oscillations in a system with a piecewise smooth coefficient, J. Sound and Vibration 283 (2005), 1229-1234.

[19] Yu. Ilyashenko, Centennial history of Hilbert's 16th problem, Bull. Amer. Math. Soc. 39 (2002), 301-354.

[20] M. Iтон And L.O. ChuA, Memristor oscillators, Int. J. Bifurcation and Chaos, vol. 18 , 3183-3206.

[21] A.I. Khibnik, B. Krauskopf and C. Rousseau, Global study of a family of cubic Liénard equations, Nonlinearity 11 (1998), 1505-1519.

[22] S. Lefschetz, Stability of Non-linear Control Systems, Academic Press, New York, 1965.

[23] J. LI, Hilbert's 16th problem and bifurcations of planar polynomial vector fields, Internat. J. Bifur. Chaos Appl. Sci. Engrg. 13 (2003), 47-106.

[24] J. Llibre, A.C. Mereu and M.A. Teixeira, Limit cycles of the generalized polynomial Liénard differential equations, Math. Proceed. Camb. Phyl. Soc. 148 (2009), 363-383.

[25] J. Llibre, M. Ordóñez And E. PONCE, On the existence and uniqueness of limit cycles in a planar piecewise linear systems without symmetry, Nonlinear Analysis Series B: Real World Applications 14 (2013), 2002-2012.

[26] J. Llibre AND E. PONCE, Three nested limit cycles in discontinuous piecewise linear differential systems with two zones, Dynamics of Continuous, Discrete and Impulsive Systems, Serie B 19 (2012), 325-335.

[27] J. Llibre and J. Sotomayor, Phase portraits of planar control systems, Nonlinear Analysis, Methods \& Applications 27 (1996), 1177-1197.

[28] J. Llibre AND A. Teruel, Introduction to the Qualitative Theory of Differential Systems, Planar, Symmetric and Continuous Piecewise Linear Differential Systems, Birkhäuser Advanced Texts, 2013.

[29] J. LlibRe AND C. VALLS, Limit cycles for a generalization of Liénard polynomial differential systems, Chaos, Solitons and Fractals 46 (2013), 65-74.

[30] K.S. Narendra And J.M TaYlor, Frequency Domain Criteria for Absolute Stability. Academic Press, New York, 1973.

[31] L. Perko, Differential equations and dynamical systems, Texts in Applied Mathematics 7, Springer-Verlag, 1991.

[32] E. Ponce, J. Ros and E. Vela, The focus-center-limit cycle bifurcation in discontinuous planar piecewise linear systems without sliding, in Progress and Challenges in Dynamical Systems (S. Ibáñez, J. S. Pérez del Río, A. Pumario and J.A. Rodríguez, editors), Proceedings of the International Conference Dynamical Systems: 100 Years after Poincaré, September 2012, Gijón (Spain), Springer Proceedings in Mathematics \& Statistics 54 (2013) 335-349.

[33] S. Smale, Mathematical Problems for the Next Century, Mathematical Intelligencer 20 (1998), 7-15.

[34] D. XIAO AND Z. ZHANG, On the uniqueness and nonexistence of limit cycles for predator-prey systems, Nonlinearity 16 (2003), 1185-1201.

[35] Ye Yan-Qian And Others, Theory of Limit Cycles, Translations of Math. Monographs, Vol. 66, Amer. Math. Soc, Providence, 1986.

[36] Z. Zhang Zhifen, T. Ding, W. Huang and Z. Dong, Qualitative Theory of Differential Equations, Translations of Math. Monographs, Vol. 101, Amer. Math. Soc, Providence, 1992.

[37] Y. Zou, T. KüPPER AND W.-J. Beyn, Generalized Hopf bifurcations for planar Filippov systems continuous at the origin, J. Nonlinear Science 16 (2006), 159-177.

1 Departament de Matemàtiques, Universitat Autònoma de Barcelona, 08193 Bellaterra, Barcelona, CATAlonia, Spain

E-mail address: jllibre@mat.uab.cat

2 Departamento de Matemática Aplicada, Escuela Técnica Superior de Ingeniería, Avda. De los Descubrimientos, 41092 Sevilla, Spain

E-mail address: eponcem@us.es 
3 Departamento de Matemática, Instituto Superior Técnico, Universidade Técnica De Lisboa, 1049-001 Lisboa, Portugal

E-mail address: cvalls@math.ist.utl.pt 